\title{
Advanced Abdominal Pregnancy at Full Term with Live Fetus Invading Colon Mesentery in a Patient with Relative Infertility, Case Report
}

\author{
Hagar G Okda ${ }^{1^{*}}$, Nadeem Hamed ${ }^{2}$, Samar Abdel Aziz ${ }^{3}$ and Ismail E Khalifa \\ ${ }^{1}$ Assistant Lecturer, Shatby University Maternity Hospital, Alexandria, Egypt \\ ${ }^{2}$ Lecturer, Shatby University Maternity Hospital, Egypt \\ ${ }^{3}$ Senior Resident, Shatby University Maternity Hospital, Beheira, Egypt \\ ${ }^{4}$ Resident, Shatby University Maternity Hospital, Alexandria, Egypt
}

*Corresponding author: Hagar G Okda, Assistant lecturer, Shatby University Maternity Hospital, Alexandria, Egypt

\begin{abstract}
Abdominal pregnancy is rare, it accounts for about $1 \%$ of ectopic pregnancies. It has a high misdiagnosis rate especially if the case is presented for the first time at advanced gestational age. In most of the published cases, abdominal pregnancies do not get to full term gestation and usually the end result is the extraction of a dead fetus or congenitally malformed live fetus. We report a case of undiagnosed advanced abdominal pregnancy at full term that was presented to our hospital for elective caesarean section. During laparotomy, it was found that she had abdominal pregnancy with placenta invading colonmesentery and left infundibula-pelvic ligament obliging us to perform left adnexectomy and Hartmann's operation. The mother and baby were healthy. We must be aware that abdominal pregnancy can be overlooked especially when the patient visits for the first time at advanced gestational age and that it can continue till term with placental invasion into the pelvic organ causing grave problems.
\end{abstract}

\section{Introduction}

Ectopic pregnancy represents about 1-2\% of all pregnancies, with $95 \%$ of those occurring in the fallopian tubes [1]. Abdominal pregnancy is a rare variant of ectopic pregnancy resulting from implantation of the embryo in the peritoneal cavity and it is termed advanced when it has gone beyond 20 weeks gestational age [2]. Abdominal pregnancy is divided into either primary pregnancy when implantation occurs directly in the abdominal cavity and secondary when extrauterine tubal pregnancy ruptures and gets re-implanted in the abdominal cavity [3]. Despite advances in imaging techniques and adequate antenatal care, cases of undiagnosed advanced abdominal pregnancies at term are still discovered accidentally intraoperatively [4]. The maternal mortality rate can be as high as $20 \%$ because of hemorrhage during placental separation as the placenta can be attached to the uterine wall, bowel, mesentery, liver, spleen, bladder and ligaments [5].

\section{Case Presentation}

The patient was 40 -years-old, married since 7 years, gravida 7 para zero abortion 6 presented to our hospital at 38 weeks +3 days gestational age for elective cesarean section because of history of previous myomectomy and being 40-year-old primipara with relative infertility for 7 years. The patient stated that she had regular antenatal care and showed an ultrasound report (Figure 1) done at gestational age of 30 weeks $+4 \mathrm{ds}$ stating normal sonographic features of the fetus with normal doppler indices of the umbilical artery and that the placenta was located fundo-anteriorly showing few hypoechoic spaces of venous lakes.

Medical history of the patient was unremarkable but her surgical history included a procedure of open myomectomy. By examination, the patient was vitally 


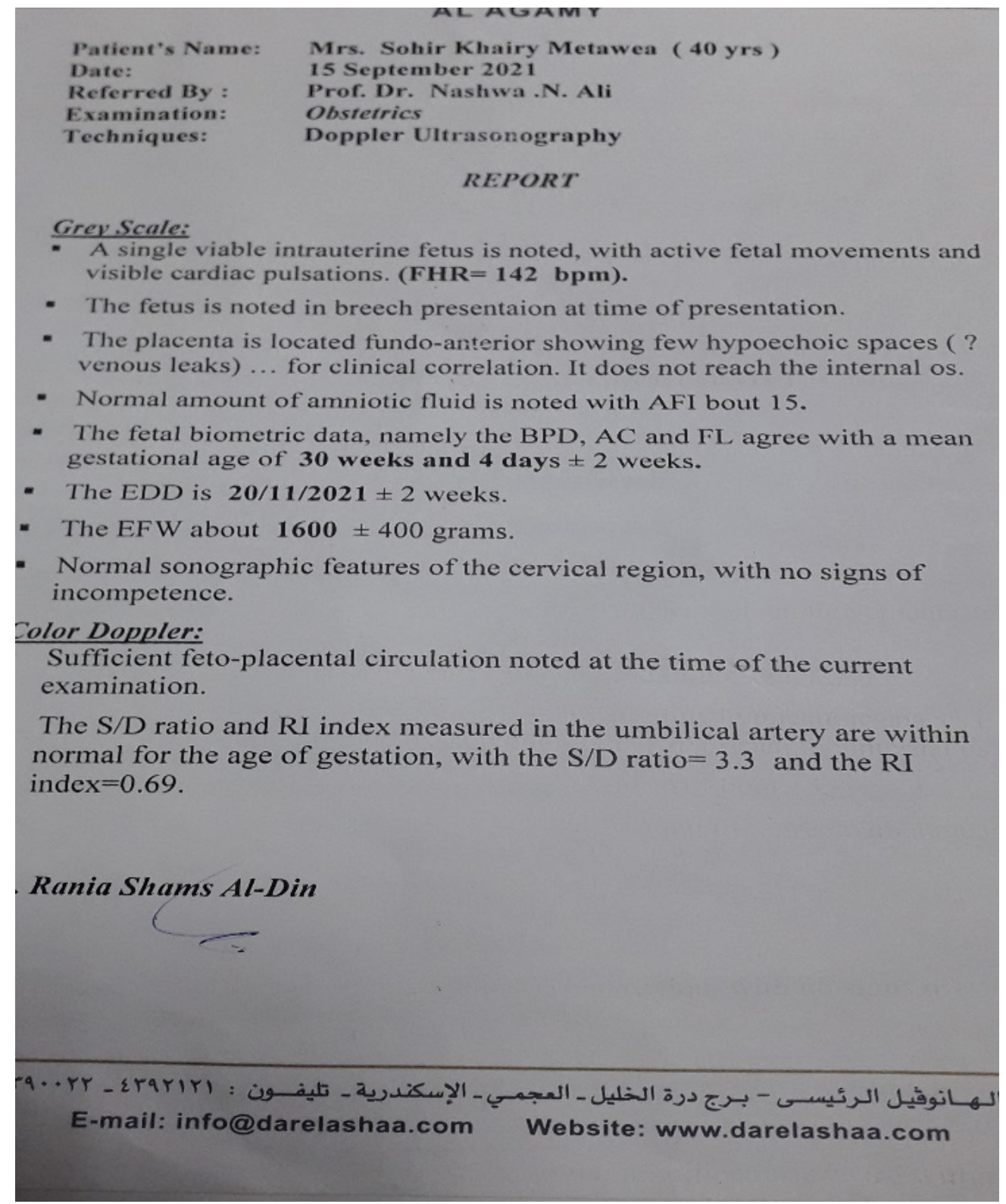

Figure 1: Doppler ultrasonography performed at the gestational age of $30 \mathrm{wks}+4 \mathrm{ds}$ stating that the placenta is located at fundal anterior position.

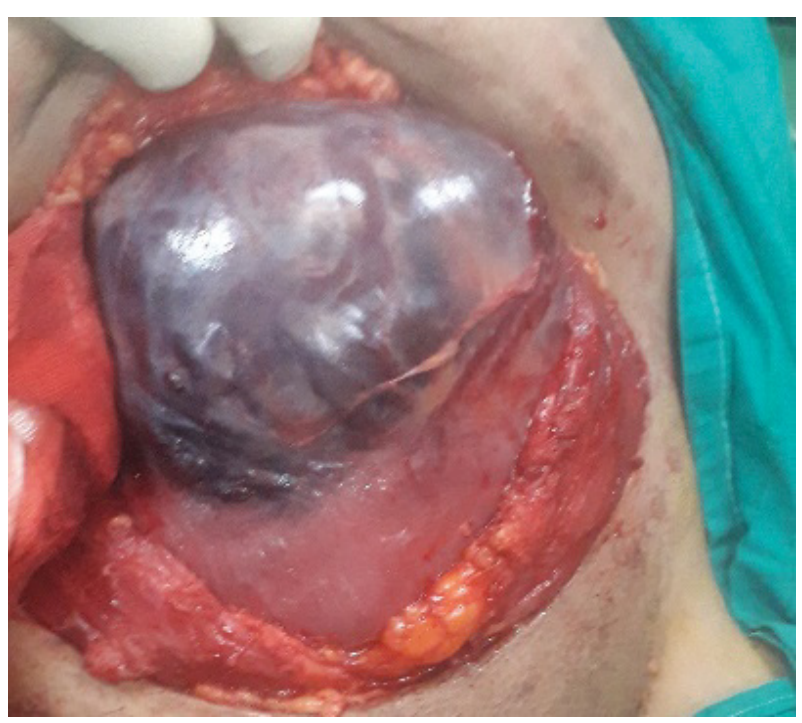

Figure 2: Empty uterus with extensive vascularity at left cornual end extending to left infundibulopelvic ligament. stable with pulse of 80 beats/min and blood pressure of $120 / 80$. Abdominopelvic examination showed fundal level of 38 weeks with no detected uterine contractions and closed cervical canal. Ultrasound was done which showed a 37 weeks of gestation viable fetus with normal heart rate at breech presentation with normal amount of amniotic fluid. In the operating room, the incision of the abdomen was done through pfannestial incision, after accessing the peritoneal cavity, the uterus showed abnormal vascularity at left broad ligament and left cornual end extending to left infundibulopelvic ligament (Figure 2).

Exteriorization of the uterus was performed and the fetus was found to be extrauterine (Figure 3 ).

Delivery of a healthy male fetus weighing 2900 gm with Apgar score of 7 and 9 in 1 and 5 minutes, respectively. 


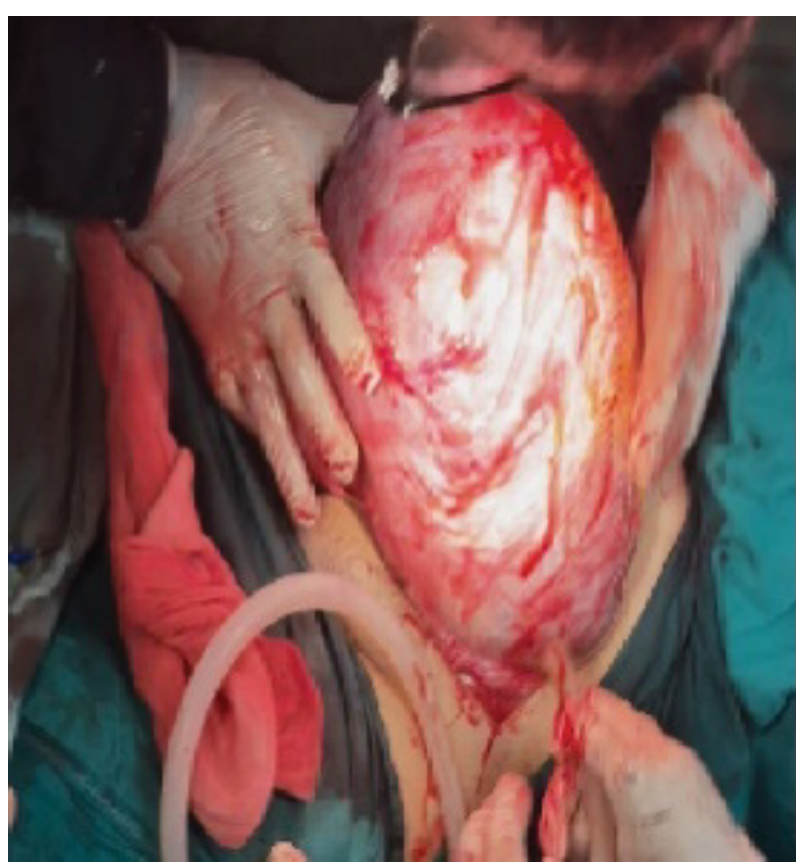

Figure 3: Exteriorization of the uterus with extrauterine sac attached to left and back of the uterus.

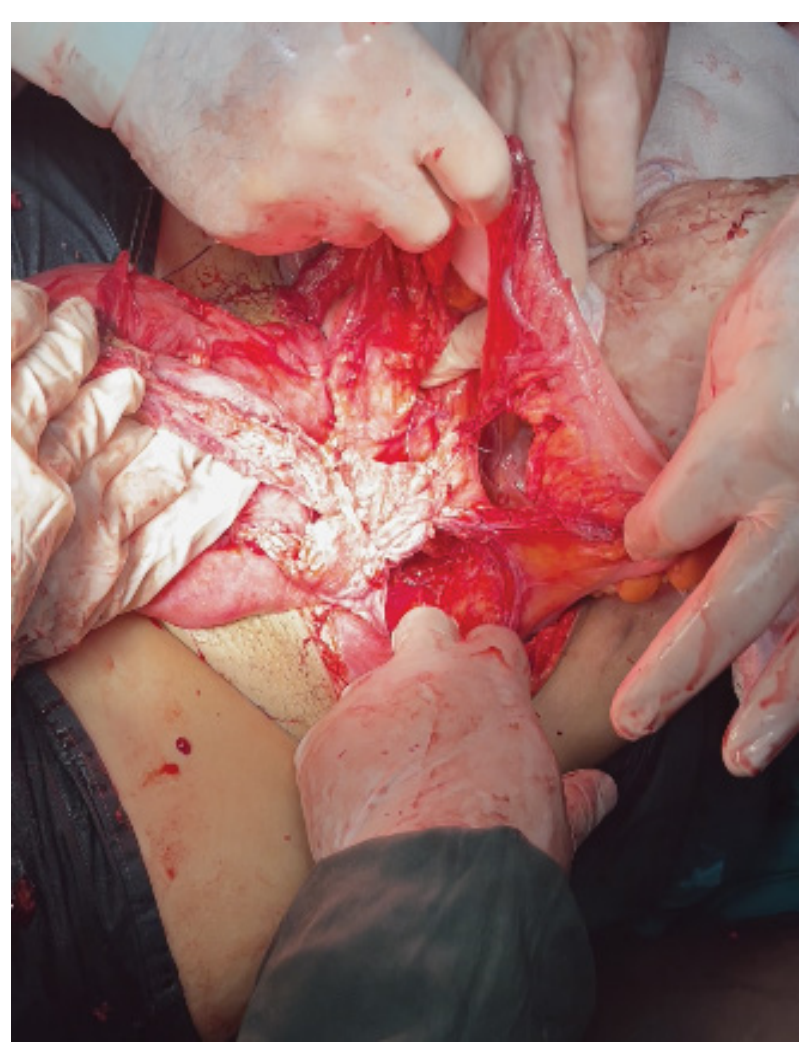

Figure 4: Started devascularization of colonic segment after inferior mesenteric artery ligation and excision of part of mesentery during placental removal.

After extraction of the fetus, placenta was found to be invading sigmoid colon mesentery and the left infundibulopelvic ligament. Removal of the placenta was done but resulted in injury to inferior mesenteric artery and left infundibulopelvic ligament.

Ligation of inferior mesenteric artery was done. Ischemia of the sigmoid colon was noted following ligation of the inferior mesenteric artery and the Hartmann's operation was performed by colorectal surgery specialist because intraoperative conditions contraindicated performance of an anastomosis (Figure 4).

Ligation of the left infundibulopelvic ligament was done followed by noted ischemia of the left ovary so left oophorectomy was performed. She was transfused with three units of blood and four units of fresh frozen plasma. Closure of the abdomen in layers after adequate haemostasis and insertion of an intraperitoneal drain. The mother was transferred to ICU postoperatively for observation for one day and following she was transferred to the ward for 48 hours and then discharged from hospital. The neonate was discharged home after 6 hours of observation in the neonatal unit. Regular check up visits for the mother were scheduled that showed no complications during puerperium and the mother was scheduled for closure of the colostomy with the colorectal surgery team. Regular check up visits were also scheduled for the baby in the neonatal unit at our hospital and were uneventful.

\section{Discussion}

Extrauterine abdominal pregnancy beyond second trimester and with a viable fetus is an extremely rare condition. Clinical diagnosis can be very difficult as there are no definite clinical signs and ultrasound is disappointing in the later stages of pregnancy [6]. Other radiological studies such as MRI and CT scan are helpful in the later stages but a high index of suspicion is required to request those imaging studies [7]. Our patient had regular antenatal care and doppler ultrasound performed without suggestion of the possibility of extrauterine pregnancy.

Complications from abdominal pregnancy include both fetal and maternal complications. Fetal complications are usually in the form of fetal death or multiple congenital anomalies [8]. Maternal complications mainly are due to abnormal placentation with placenta attached to vital highly vascular structures. Placenta could be removed during laparotomy, however this maneuver has its own risks due to expected massive haemorrhage and need of removal of the organ or the structure to which the placenta is attached to control bleeding [9]. Alternatively, some authors recommended to leave the placenta in situ and monitor the patient's human chorionic gonadotropin levels [10]. The use of methotrexate to accelerate the resorption is controversial as it might involve a greater risk of infection [11].

In our case, placenta was attached to colon mesentery and left infundibulopelvic ligament and its separation resulted in inferior mesenteric artery injury with subsequent Hartmann's procedure and left oophorectomy. For the newborn, it is very important 
to rule out congenital malformations. There are reports of fetal malformations as high as $40 \%$ associated with abdominal pregnancies [12]. In this case no congenital malformations were detected and the baby showed normal morphological appearance and reflexes on examination and was discharged home as a well baby.

\section{Conclusion}

Abdominal pregnancy which results in a live newborn at full term without congenital anomalies is very rare. Abdominal pregnancy poses diagnostic challenges with ultrasound accuracy of $50 \%$ [13]. Without a high index of suspicion the diagnosis will be missed, only to be made during laparotomy. The decision whether to remove the placenta or leave it in situ is the most challenging in cases of advanced abdominal pregnancy.

\section{Acknowledgements}

We acknowledge the staff of Shatby Maternity University Hospital who participated in the care of this patient.

\section{Competing Interests}

The authors declare no competing interests.

\section{Authors' Contributions}

All the authors shared in the operation and followed the patient postoperatively. All the authors have read and agreed to the final manuscript.

\section{References}

1. Nwobodo El (2004) Abdominal pregnancy: A case report. Ann Afr Med 3: 195-196.

2. Nassali MN, Benti TM, Bandani-Ntsabele M, Musinguzi E (2016) A case report of an asymptomatic late term abdominal pregnancy with a live birth at 41 weeks of gestation. BMC Res Notes 9: 31.
3. Singh Y, Singh SK, Ganguly M, Singh S, Kumar P (2016) Secondary abdominal pregnancy. Med J Armed Forces India 72: 186-188.

4. Huang K, Song L, Wang L, Gao Z, Meng Y, et al. (2014) Advanced abdominal pregnancy: An increasingly challenging clinical concern for obstetricians. Int J Clin Exp Pathol 7: 5461-5472.

5. Yildizhan R, Kolusari A, Adali F, Adali E, Kurdoglu M, et al. (2009) Primary abdominal ectopic pregnancy: A case report. Cases J 2: 8485.

6. Worley KC, Hnat MD, Cunningham FG (2008) Advanced extrauterine pregnancy: Diagnostic and therapeutic challenges. Am J Obstet Gynecol 198: 297.

7. Chitra KL (2007) Viable abdominal pregnancy. J Obstet Gynaecol India 57: 169-170.

8. Nkusu Nunyalulendho D, Einterz EM (2008) Advanced abdominal pregnancy: Case report and review of 163 cases reported since 1946. Rural Remote Health 8: 1087.

9. Varma R, Mascarenhas L, James D (2003) Successful outcome of advanced abdominal pregnancy with exclusive omental insertion. Ultrasound Obstet Gynecol 21: 192-194.

10. Badria L, Amarin Z, Jaradat $A$, Zahawi $H$, Gharaibeh $A$, et al. (2003) Full-term viable abdominal pregnancy: A case report and review. Arch Gynecol Obstet 268: 340-342.

11. Riethmuller D, Courtois L, Maillet R, Schaal JP (2003) Ectopic pregnancy management: Cervical and abdominal pregnancies. J Gynecol Obstet Biol Reprod (Paris) 32: S101-S108.

12. Baffoe P, Fofie C, Gandau BN (2011) Term abdominal pregnancy with healthy newborn: A case report. Ghana Med J 45: 81-83.

13. Agarwal N, Odejinmi F (2014) Early abdominal ectopic pregnancy: Challenges, update and review of current management. Obstet Gynaecol 16: 193-198. 